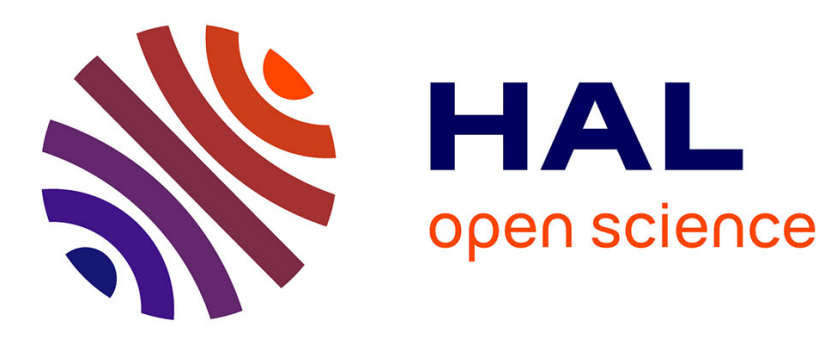

\title{
Ferrofluid Leidenfrost droplets
}

Christophe d'Angelo, Christophe Raufaste, Pavel Kuzhir, Franck Celestini

\section{To cite this version:}

Christophe d'Angelo, Christophe Raufaste, Pavel Kuzhir, Franck Celestini. Ferrofluid Leidenfrost droplets. Soft Matter, 2019, 15 (29), pp.5945-5950. 10.1039/c9sm00724e . hal-02426110

\section{HAL Id: hal-02426110 https://hal.science/hal-02426110}

Submitted on 6 Jan 2020

HAL is a multi-disciplinary open access archive for the deposit and dissemination of scientific research documents, whether they are published or not. The documents may come from teaching and research institutions in France or abroad, or from public or private research centers.
L'archive ouverte pluridisciplinaire HAL, est destinée au dépôt et à la diffusion de documents scientifiques de niveau recherche, publiés ou non, émanant des établissements d'enseignement et de recherche français ou étrangers, des laboratoires publics ou privés. 


\author{
Christophe D’Angelo, ${ }^{a}$ Christophe Raufaste, ${ }^{a}$ Pavel Kuzhir,${ }^{a}$ and Franck Celestini ${ }^{* a}$
}

Received Date

Accepted Date

www.rsc.org/journalname
DOI: 10.1039/xxxxxxxxxx

\begin{abstract}
We experimentally investigate the behavior of ferrofluid Leidenfrost droplets subject to a static magnetic field gradient. The droplets are deposited on a hot substrate and trapped over the vertical axis of a permanent magnet placed at a distance $d$ above the substrate. Several effects are observed. Firstly, the droplet evaporation rate is strongly influenced by the distance $d$. Secondly, the droplet takes off from the substrate when its radius decreases to a critical value. The introduction of an effective gravity, which accounts for the magnetic force, allows a successful description of these effects. Finally, we observe an instability for which the droplet starts bouncing with irregular amplitudes. This behavior is qualitatively interpreted by introducing the synchronization of the free fall time between successive bounces with the period of the fundamental vibration mode of the droplet.
\end{abstract}

\section{Introduction}

When a droplet is deposited on a substrate heated at a sufficiently high temperature, typically $100^{\circ} \mathrm{C}$ higher than its boiling temperature, it levitates on its own vapor without any contact between the liquid and the supporting substrate. Such droplets, called Leidenfrost droplets ${ }^{1}$, are easily set into motion as compared to droplets deposited on hydrophobic substrates that are subject to wetting angle hysteresis ${ }^{2,3}$. As a consequence, numerous studies have evidenced striking dynamical properties ${ }^{4}$ that are interesting for fundamental research as well as for possible applications in the field of droplet actuation. As examples we can cite the self-propulsion of droplets under a critical size ${ }^{5}$ or on anisotropic substrates ${ }^{6,7}$, the take-off of small droplets ${ }^{8}$ and the spontaneous destabilization of quasi-2D droplet in a Hele-Shaw cell $^{9-11}$.

Recently Piroird et al. ${ }^{12}$ have used magnetic fields to trap and manipulate paramagnetic oxygen droplets in the Leidenfrost state. In the same vein we propose here to enhance magnetic effects by using ferrofluid Leidenfrost droplets.

We will show that the dynamics of a ferrofluid droplet reveals peculiar aspects both quantitatively and qualitatively different from the ones observed with a paramagnetic fluid. First, the evaporation rate depends strongly on the substrate-magnet distance. Second, the droplet does not remain immobile but exhibits a complex dynamics. The droplet bounces spontaneously and finally takes off to reach the magnet. Such aspects have similarities with Leidenfrost droplets trapped by an electrical field ${ }^{13}$. We finally

\footnotetext{
${ }^{a}$ Université Côte d'Azur, CNRS UMR 7010, Institut de Physique de Nice, Parc Valrose, 06100 Nice, France

$\dagger$ Electronic Supplementary Information (ESI) available: movie used to build the space-time diagram of Fig. 3. See DOI: 10.1039/cXsm00000x/

*Corresponding author: Franck.Celestini@unice.fr
}

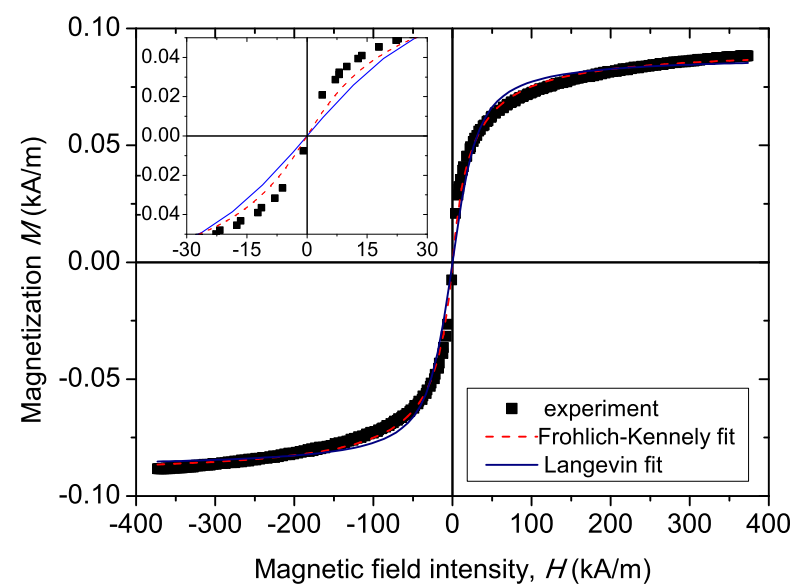

Fig. 1 Magnetization curve of the ferrofluid. The inset shows a magnified view centered around the origin.

show that the features of the bounces can be described using an effective gravity and the synchronization between the time scales of the free falls between successive bounces and the period of the fundamental vibration mode of the droplet.

\section{Materials and methods}

\subsection{Ferrofluid synthesis and characterization}

A ferrofluid containing iron oxide nanoparticles dispersed in distilled water and stabilized by a double layer of oleate ions was synthesized using well-known co-precipitation method described in Bica et al. ${ }^{14}$. During synthesis, iron oxide nanoparticles of an average size $8 \mathrm{~nm}$ were associated to form permanent quasispherical nanoclusters of an average size $50 \mathrm{~nm}$. Typical transmission electron microscope (TEM) images of these nanoclusters 
are shown in Ref. ${ }^{15}$. The particle volume fraction $\Phi$, the particle density, and the ferrofluid density are respectively $2.8 .10^{-4}, 5000$ $\mathrm{kg} \cdot \mathrm{m}^{-3}$ and $997 \mathrm{~kg} \cdot \mathrm{m}^{-3}$ at room temperature.

Magnetization of the ferrofluid $M$ versus applied magnetic field intensity $H$ (so-called magnetization curve) was measured by a vibrating sample magnetometer VSM 4500 (EG\&G Princeton applied Research, United States) at ambient temperature. The measured magnetization curve is shown in Fig. 1 and it does not exhibit any hysteresis confirming the superparamagnetic behavior of the ferrofluid.

The magnetization curve was first fitted by the Langevin law ${ }^{16}$, which can be written in the following form: $M=$ $M_{S}\left(\operatorname{coth}\left(H / H^{*}\right)-H^{*} / H\right)$, where $M_{S}$ is the ferrofluid saturation magnetization and $H^{*}$ is the value of the magnetic field intensity at which the magnetic energy of the interaction between the ferrofluid nanoclusters and the applied magnetic field is equal to the thermal agitation energy. As inferred from Fig. 1, the Langevin law does not perfectly fit the magnetization curve. This can be explained by the fact that the magnetization mechanism of the ferrofluid composed of multicore nanoclusters is much more complicated than the one of individual single-domain nanoparticles $^{17}$. Since the precise magnetization mechanism of a ferrofluid composed of nanoclusters is still not completely established, it is reasonable to fit the magnetization curve by an empirical magnetization law, such as the Fröhlich-Kennely one ${ }^{18}$,

$$
M=\chi M_{S} H /\left(\chi H+M_{S}\right),
$$

with two adjustable parameters: $M_{S}$ - the saturation magnetization and $\chi$ - the initial magnetic susceptibility . As shown in Fig. 1, the Fröhlich-Kennely law (solid blue line) gives a better interpolation of the experimental magnetization curve than the Langevin law (dashed red line) and will be used below for calculations of ferrofluid droplets magnetization. We obtained the following values of the adjustable parameters: $\chi=4.15 .10^{-3} \pm 0.50 .10^{-3}$ and $M_{S}=9 \cdot 18 \cdot 10^{-2} \pm 0.03 \cdot 10^{-2} \mathrm{kA} \cdot \mathrm{m}^{-1}$.

Since Leidenfrost droplets were heated up to the boiling temperature, about $100{ }^{\circ} \mathrm{C}$, the particle volume fraction and ferrofluid density as well as the droplet magnetization have to be re-evaluated. Firstly, the density of water at $100^{\circ} \mathrm{C}$ is $958 \mathrm{~kg} \cdot \mathrm{m}^{-3}$, slightly lower than at room temperature. The density of the nanoparticles is almost the same as the one at room temperature. Since the particle volume fraction at room temperature is very small $\left(\Phi \simeq 2.8 .10^{-4}\right)$, it is easily checked that the particle volume fraction at $100^{\circ} \mathrm{C}$ should remain almost the same while the ferrofluid density becomes $\rho_{f}=959 \mathrm{~kg} \cdot \mathrm{m}^{-3}$. Secondly, as the drop evaporates on the heated substrate, its mass progressively decreases with time. However, in the present case of low particle volume fraction $\Phi \simeq 2.8 \cdot 10^{-4} \ll 1$, the ferrofluid density variation during droplet evaporation is negligible with respect to the volume variation of the droplet. The ferrofluid density is therefore assumed to be constant and equal to its initial value. Finally, the droplet magnetization at a given magnetic field intensity $H$ was re-evaluated assuming that the droplet magnetization saturation, $M_{S}$, was independent of temperature, while the initial magnetic susceptibility, $\chi$, was inversely proportional to the absolute

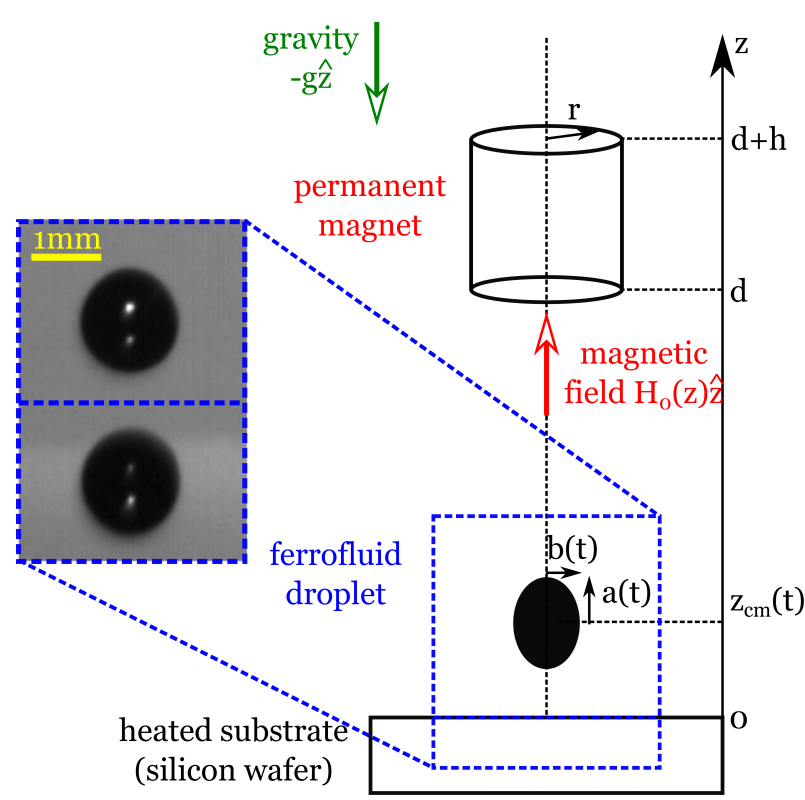

Fig. 2 Experimental setup. The picture shows the drop and its mirror image in the silicon wafer.

temperature $T$. Thus, $M(T)$ at boiling temperature $T$ was evaluated using the Fröhlich-Kennely law by replacing $\chi$ by $\chi\left(T_{0}\right) T_{0} / T$, where $T_{0}$ is the room temperature.

\subsection{Experimental setup and protocol}

The experimental setup is depicted in Fig. 2. Droplets are deposited on a silicon wafer heated at $T=220^{\circ} \mathrm{C}$. The droplet volume is controlled with a micropipette so that the initial droplet radius is kept constant through the whole study, $R_{0}=1.22 \pm 0.13$ $\mathrm{mm}$. A cylindrical neodymium magnet (height $h=10 \mathrm{~mm}$, radius $r=5 \mathrm{~mm}$, magnetic flux density on the surface $\mu_{0} M_{\text {magnet }}=1.3 \mathrm{~T}$ with $\mu_{0}=4 \pi \cdot 10^{-7} \mathrm{H} \cdot \mathrm{m}^{-1}$ the magnetic permeability of vacuum) is fixed above the substrate with its axis aligned with the vertical dimension. The distance $d$ between the bottom of the magnet and the substrate is varied between 2.5 and $12 \mathrm{~mm}$. The droplet is trapped along the axis of the magnet and its horizontal position does not evolve with time.

As reported below the coupling between gravity and the magnetic force gives a complex dynamics. A space-time diagram, built along the vertical line passing through the center of the droplet (Figure 3), illustrates that the droplet bounces until it is finally trapped by the magnet. During a free fall between two bounces its shape evolves and is well fitted by the one of an ellipsoid of revolution along the $z$-axis as illustrated in Fig. 2 .

Quantitative measurements are performed by tracking the droplet with a high-speed camera at a frame rate of 4000 fps. At each time step the center of mass $z_{\mathrm{cm}}(t)$ is recorded and the lateral projection of the droplet is fitted by an ellipse. The two axes of the ellipse, $a(t)$ along the vertical axis and $b(t)$ along the horizontal axis, are recorded. The effective radius of the droplet is inferred by $R(t)=a(t)^{1 / 3} b(t)^{2 / 3}$. 


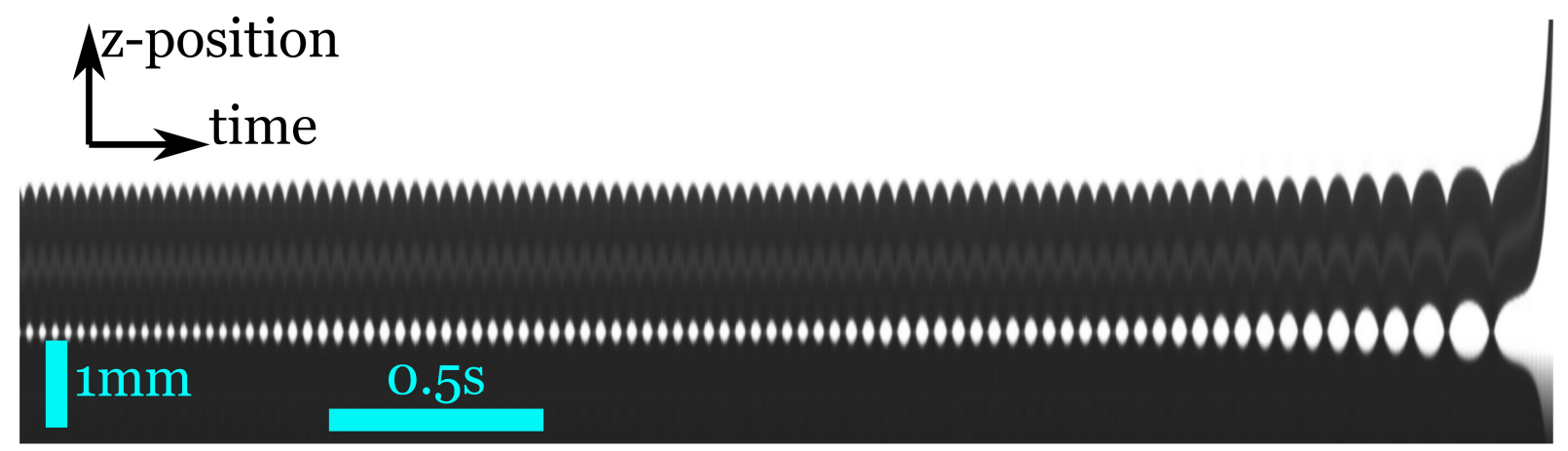

Fig. 3 Space-time diagram along the $z$ axis passing through the center of the droplet and its mirror image on the silicon wafer (movie in the supplementary material $\dagger$ ). The last 3.5 seconds are displayed and illustrate the multiple bounces that occur before the final take-off. In this experiment $d=6.1 \mathrm{~mm}$.

\subsection{Magnetic field}

As indicated above, the external magnetic field is provided by a cylindrical neodymium magnet (radius $r$ and height $h$ ) located at a distance $d$ above the substrate (Fig. 2). The magnetic field $H_{d}(z)$ along the $z$-axis of the cylinder is given by:

$$
H_{d}(z)=\frac{M_{\text {magnet }}}{2}\left(\frac{h+d-z}{\sqrt{(h+d-z)^{2}+r^{2}}}-\frac{d-z}{\sqrt{(d-z)^{2}+r^{2}}}\right) .
$$

where the subscript $d$ in $H_{d}$ emphasizes that $d$ is a parameter in the expression of the magnetic field. The magnetic field is an increasing function of the position $z$, from the surface of the substrate $(z=0)$ to the bottom of the magnet $(z=d)$. This expression, as well as the value of the magnet magnetization, was checked by measuring the magnetic field distribution using a gaussmeter Caylar GM-H103 (France).

We assume that the magnetic field is homogeneous at the scale of the drop and is equal to the magnetic field generated by the magnet in the absence of the drop at a position corresponding to the drop center of mass $z_{c m}(t)$. The drop is trapped along the $z$ axis so that the field experienced by the drop is given by Eq. 2 . As discussed in 2.1, the diluted ferrofluid assumption gives the following magnetic force experienced by the drop:

$$
F_{M}\left(z_{c m}\right)=\mu_{0} \mathscr{M}\left(H_{d}\left(z_{c m}\right)\right) \frac{d H_{d}}{d z}\left(z_{c m}\right),
$$

where the magnetic moment of the droplet is $\mathscr{M}\left(H_{d}\right)=$ $\frac{4 \pi R(t)^{3}}{3} M\left(H_{d}\right)$. It is important to note that since the number of magnetic particles in the ferrofluid droplet remains the same, as the suspending liquid evaporates, the magnetic moment of the drop is constant over the time and can be related to the initial magnetization and initial radius of the droplet through the following expression: $\mathscr{M}\left(H_{d}\right)=\frac{4 \pi R_{0}^{3}}{3} M_{0}\left(H_{d}\right)$. Notice that in the expression of $F_{M}$ (Eq. 3) and in this last expression of $\mathscr{M}\left(H_{d}\right)$ we have neglected the demagnetizing field in the ferrofluid droplet, $-M\left(H_{d}\right) / 3$, since it almost vanishes in the present case of diluted magnetic droplets with $M\left(H_{d}\right) \ll H_{d}$, as inferred from the magnetization curve (Fig. 1).

To account for both magnetic and gravity effects in the net bulk force, we define an effective gravity as $-m g_{\text {eff }}=P+F_{M}$, where $P(t)=-m(t) g=-\rho_{f}(t) \frac{4}{3} \pi R(t)^{3} g$ is the droplet weight at time $t$.
This leads to :

$$
g_{e f f}(t)=g-\frac{\mu_{0}}{\rho_{f}}\left(\frac{R_{0}}{R(t)}\right)^{3} \mid M_{0}\left(H_{d}\left(z_{c m}(t)\right) \frac{d H_{d}}{d z}\left(z_{c m}(t)\right) \mid .\right.
$$

It is worth noting that $g_{\text {eff }}(t)<g$ because of the presence of the magnetic force. The time dependency of the effective gravity comes from the dynamical evolution of the droplet radius and its position above the substrate.

\section{Results}

Initially the droplet rests on the heated substrate and is trapped along the main axis of the magnet. The dynamics of this Leidenfrost ferrofluid droplet exhibits several aspects that are described in the following subsections:

1. The evaporation rate depends strongly on the distance between the droplet and the magnet.

2. We observe spontaneous bounces of the droplet subject to an effective gravity.

3. The droplet takes off and reaches the magnet at a certain critical size.

4. The bouncing dynamics exhibits a non-monotonous behavior with the occurrence of boosts that increase suddenly the amplitude of the bounces.

\subsection{Evaporation rate}

Figure 4 displays the evaporation dynamics as a function of the magnet-substrate distance $d$. All droplets have the same initial radius $R_{0}$. We observe that the larger the distance $d$, the faster the evaporation. Actually the decrease of the radius with time is almost linear and we can define an average evaporation rate $\alpha=-(d R / d t)$ as seen in the inset of Fig. 4. The latter is an increasing function of the distance $d$ and varies over a factor 3 within our experimental conditions.

This result is in good agreement with previous studies of evaporation rates. First, for large values of $d$ we checked that the evaporation rates are consistent with typical values obtained in standard gravity condition ${ }^{20,21}$. Second, in Maquet et al. ${ }^{22}$ droplets are shown to evaporate quicker in high gravity conditions. In 


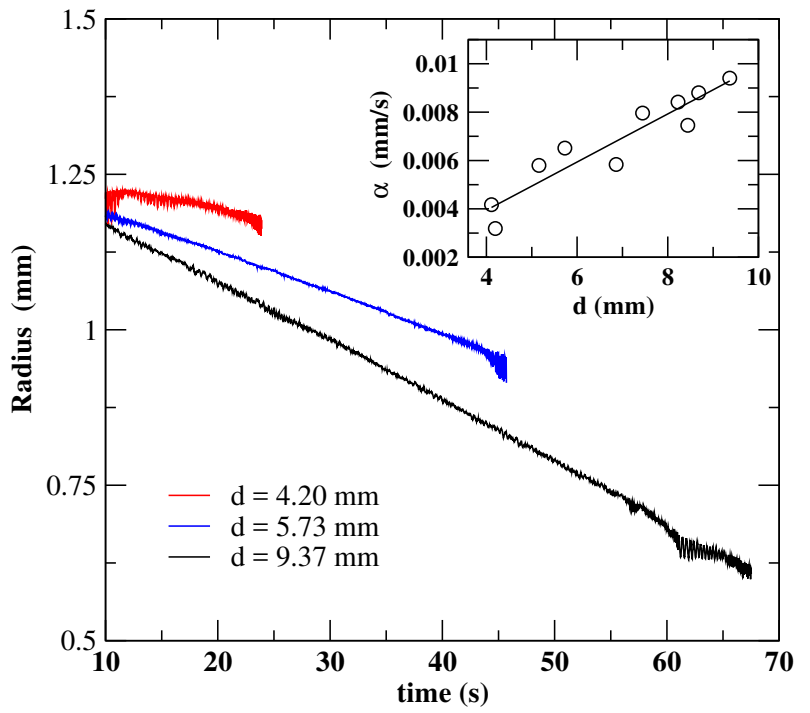

Fig. 4 Dynamical evolution of the droplet radius for experiments performed with different distances $d$ between the substrate and the magnet. Inset : evaporation rate $\alpha=-d R / d t$ as a function of the substrate-magnet distance $d$. The linear interpolation, solid line, is a guide for the eye.

our case, as the magnet-substrate distance decreases, the effective gravity is lowered, which is therefore consistent with the evaporation rate measurements presented just above.

Decreasing the evaporation rate does not mean that the lifetime of the droplet on the substrate is increased. As seen below, the droplets take off for a critical radius and the smaller $d$, the larger this radius. As a consequence, while evaporation rates are smaller for smaller $d$, droplets take off quicker. The largest lifetimes "on the substrate" are thus obtained for the largest $d$.

\subsection{Bounces}

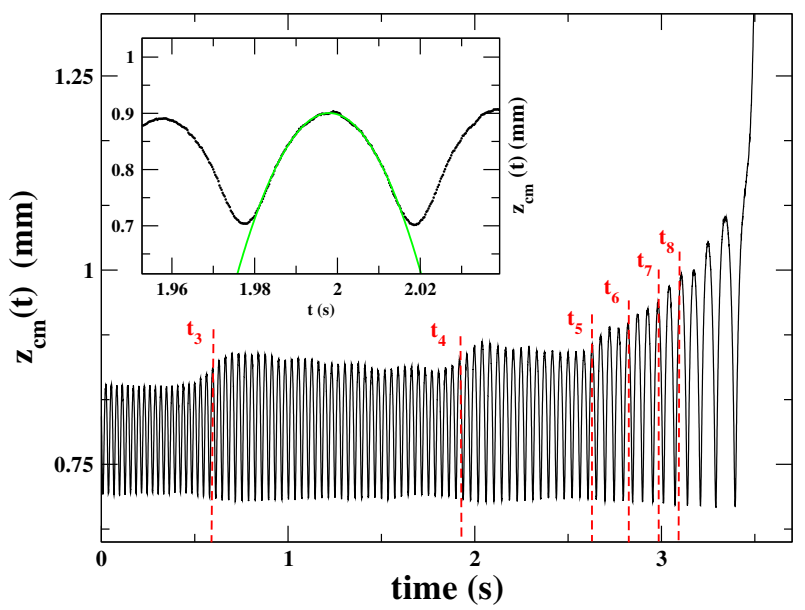

Fig. 5 Dynamical evolution of the droplet center of mass. The droplet experiences successive bounces. The general trend is that, as it evaporates, the droplet is bouncing higher until it finally takes off to reach the magnet. Note that the trend is not monotonous. The amplitude of the bounces seems to increase abruptly around specific times $\left(t_{3}, t_{4}, \ldots\right)$ followed by period of gentle decrease of the amplitude. Inset : zoom over the free fall between two bounces. The trajectory of the center of mass is successfully interpolated by a parabolic trend (light green curve).
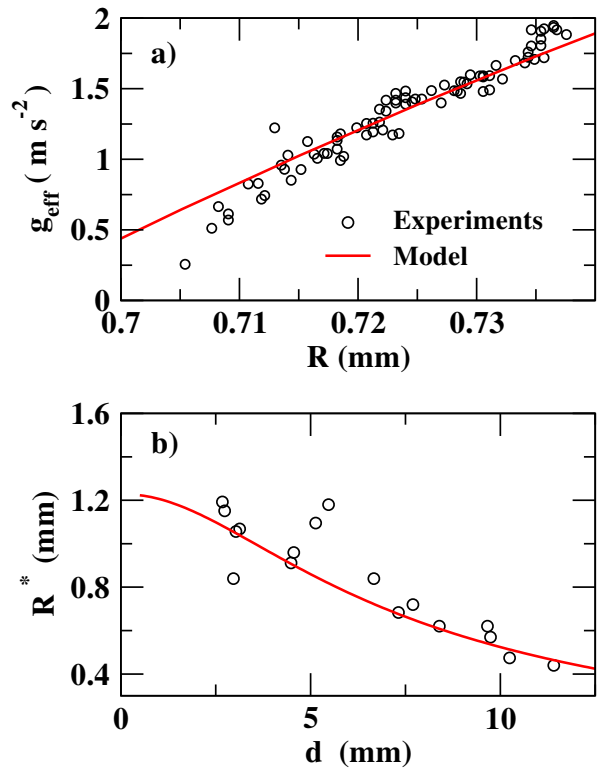

Fig. 6 a) Effective gravity as a function of the droplet radius (one point is one free fall between successive bounces on the substrate in Fig. 5). The effective gravity is inferred by fitting the position of the center of mass with a parabolic curve. The light red line represents the model (Eq. 4 with $\left.z_{c m}(t)=0\right)$. b) Critical radius at which the droplet takes off and reaches the magnet as a function of the substrate-magnet distance $d$. The light red line represents the model (Eq. 5 with $z_{c m}=0$ ).

As evidenced experimentally, the droplet bounces spontaneously and continuously on the substrate (Figure 5). The bounces are initially barely visible, but their amplitude increases significantly during the last seconds before the droplet takes off. Such a behavior was already observed with Leidenfrost droplets subject to an electrical field ${ }^{13}$. The free falls between successive bounces exhibit parabolic trajectories (inset of fig. 5). Therefore each free fall can be described by a constant effective gravity $g_{e f f}$, which remains almost constant between two bounces, although it varies over larger time scales.

This is accounted for by Eq. 4 given that, during each free fall, the drop radius does not vary significantly with time and that the fall amplitude remains small compared to the distance $d$ so that $z_{c m(t)} \simeq 0$ in Eq. 4. This last condition (small amplitude hypothesis) means that the droplet experiences a magnetic force equal to the one in $z=0$. With these assumptions the effective gravity is a function of $R$ only for a given experiment. The expression of Eq. 4 is tested in Fig. 6a and there is a very good agreement between the data and the model without any free parameter. Note that as $R$ decreases, the amplitude of the bounces increases and we can see a slight deviation from the model for the smallest radii: the small amplitude hypothesis becomes questionable.

\subsection{Final take-off analysis}

The amplitude of the magnetic force remains almost constant while the amplitude of the weight decreases due to the loss of mass. As a consequence the effective gravity decreases with time and the droplet finally takes off to reach the magnet. This occurs at the threshold, $g_{\text {eff }}=0$, that gives a relation between the droplet radius at take-off, denoted hereinafter $R^{*}$, and droplet po- 
sition $z_{c m}$ :

$$
R^{*}=R_{0}\left(\frac{\mu_{0}}{\rho_{f} g}\left|M_{0}\left(H_{d}\left(z_{c m}\right)\right) \frac{d H_{d}}{d z}\left(z_{c m}\right)\right|\right)^{1 / 3} .
$$

As a first approximation we assume $z_{c m}=0$ (small amplitude hypothesis) and we expect that $R^{*} / R_{0}$ is a function of $d$ only through the expression of the magnetic field (Eq. 2). 17 experiments were performed by varying the magnet-substrate distance $d$ (2.5$12 \mathrm{~mm}$ ). The model gives a relatively good agreement without any free parameter (Fig. 6b). In general, the model slightly underestimates the take-off radii measured experimentally. In reality the effective gravity is not homogeneous in space and we underestimate the magnetic force experienced by the droplet by considering $z_{c m}=0$ : the droplet takes off during its last free fall, which has a relatively large amplitude and experiences a slightly larger magnetic force.

\subsection{Boost and synchronization analyses}
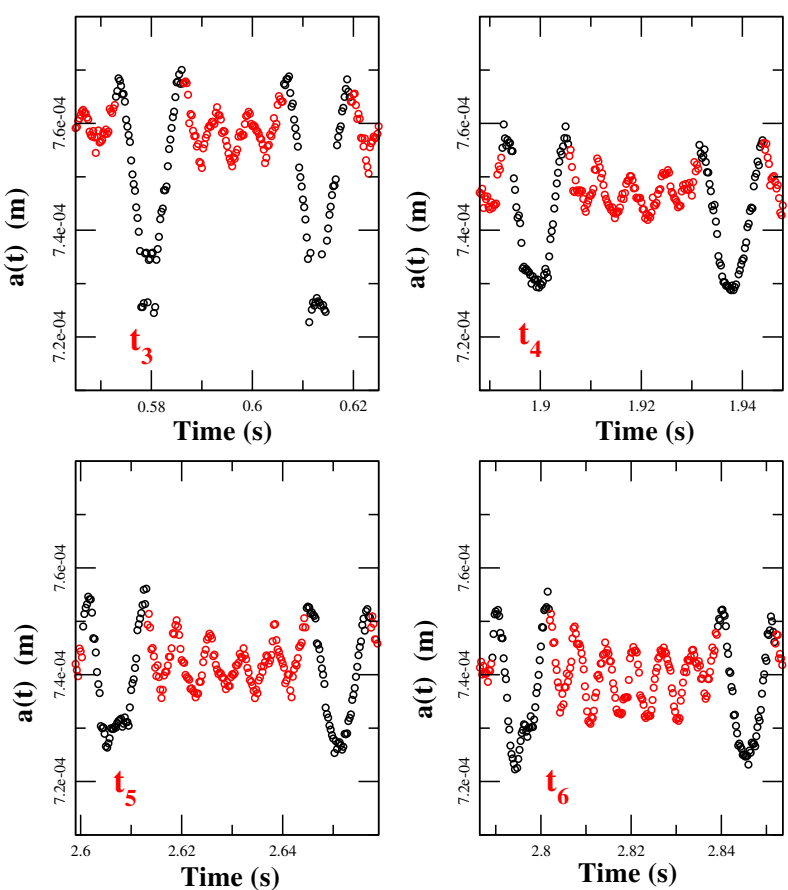

Fig. 7 Vertical axis of the droplet, $a(t)$, as a function of time around four specific times emphasized in Fig. 5 . The four snapshots stand for $t_{3}, t_{4}, t_{5}$ and $t_{6}$. Black and light red points represent the droplet status: in contact with the substrate and in free fall respectively.

From the analysis of the position of the center of mass, hundreds of bounces can be observed with the same drop although we focus on the last 90 bounces in Fig. 5. Each rebound is characterized by an impact velocity $V$ (the one when the drop contacts the substrate) and a Weber number $W e=\rho_{f} V^{2} R / \gamma$, with $\gamma$ the surface tension. We found that $W e$ is around 0.01 in our experiments, a value significantly lower than unity. As previously studied in the context of bouncing Leidenfrost droplets, Biance et al. ${ }^{23}$ found that the restitution coefficient is very close to one in this capillary dominated regime. Also, due to the magnetic effect and to the decrease of radius with time, the effective gravity experienced by the droplet is decreasing with time too. As a consequence, we should expect that the droplet bounces higher and higher before taking-off. Looking at Fig. 5, it is clear that the amplitude of the bounces is not a monotonously increasing function of time. The droplet is subject to short periods of boosts, which increase suddenly the amplitude after a few bounces. These boosts can be easily seen in Fig. 5 at time denoted $t_{3}, t_{4} \ldots$ They are followed by long periods of slow decrease of the amplitude before the next boosts. To understand this effect we look at the dynamical evolution of the vertical axis of the drop (Fig. 2). In Fig. 7, $a(t)$ is plotted around specific times described above and is displayed with two different colors to emphasize the time spent in contact with the substrate and the time spent in free fall. For all the boosts recorded, the vertical axis is maximal when the droplets are taking off as well as when they are landing back on the substrate. Biance et al. ${ }^{23}$ also shed light on an interplay between the shape of the droplet at impact and the restitution coefficient. It is clear in our case that landing and taking-off with on oblate shape gives a favorable bouncing condition. As a direct consequence there is a precise synchronization between the free fall time and the period of oscillation of the droplet. With our notation, $t_{n}$ means that the droplets perform exactly $n$ oscillations during its free fall. In Fig. 5 we have displayed these times, from $t_{3}$ to $t_{8}$, and have observed that they match exactly the period of boosts of the amplitude. We can thus interpret the irregular amplitude of the bounces as a direct consequence of this synchronization. This occurence of boosts has been recently observed for Leidenfrost droplets subject to a static electrical field ${ }^{13}$ as well. We expect that it could be related to the same synchronisation effect.

\section{Conclusion}

In this paper we have studied the behavior of ferrofluid Leidenfrost droplets deposited on a substrate and subject to a magnetic field imposed by a permanent magnet situated above. We have shown that the evaporation rate and the motion of the droplets are functions of the substrate-magnet distance. First, in our parameters range, the evaporation rate can vary by a factor 3 . Second, the droplets do not remain immobile but exhibit multiple bounces with irregular amplitudes before they take off and reach the magnet. This aspect is linked to the negligible energy loss in this system, a reduced gravity and a coupling with the oscillation modes of the droplets.

As direct applications, we can now use the magnetic field to control the evaporation rate and the lifetime of droplets. Also the good agreement between the experiments and the model concerning the existence of a critical take-off radius could be used to collect ferrofluid droplets of desired size through this magneticinduced vertical actuation.

As a perspective, the situation where the magnet is placed below the substrate could be investigated. In this case we can imagine that the magnetic field could be used to control and suppress the Leidenfrost state as recently investigated with an electric field ${ }^{24}$. 


\section{Acknowledgments}

We are grateful to T. Frisch for helpfull discussions and J. Alves Marins for her precious help with the ferrofluid synthesis.

\section{References}

1 J. G. Leidenfrost, De Aquae Communis Nonnullis Qualitatibus Tractatus (Duisbourg, 1756)

2 D. Quéré, Rep. Prog. Phys. 11, 2495 (2005).

3 D. Quéré and M. Reyssat, Phil. Trans. R. Soc. A 366, 15391556 (2008).

4 D. Quéré, Ann. Rev. Fluid. Mech. 15, 197 (2013).

5 A. Bouillant, T. Mouterde, P. Bourrianne, A. Lagarde, C. Clanet and D. Quéré, Nature Physics 14, 1188 (2018).

6 H. Linke, B. J. Alemán, L. D. Melling, M. J. Taormina, M. J. Francis, C. C. Dow-Hygelund, V. Narayanan, R. P. Taylor and A. Stout, Phys. Rev. Lett. 96, 154502 (2006).

7 T. R. Cousins, R. E. Goldstein, J. W. Jaworski and A. I. Pesci, J. Fluid. Mech. 696, 215-227 (2012).

8 F. Celestini, T. Frisch and Y. Pomeau, Phys. Rev. Lett. 109, 34501 (2012).

9 F. Celestini, T. Frisch, A. Cohen, C. Raufaste, L. Duchemin and Y. Pomeau, Phys. fluids 26, 032103 (2014).

10 C. Raufaste, F. Celestini, A. Barzyk and T. Frisch, Phys. fluids 27, 031704 (2015).

11 P. S. Raux, G. Dupeux, C. Clanet and D. Quéré, Europhys. Lett. 112, 26002 (2015).

12 K. Piroird, B. Darbois Texier, C. Clanet and D. Quéré, Phys. Fluids 25, 032108 (2013).

13 S. Wildeman and C. Sun, Soft Matter 12, 9622-9632 (2016).

14 D. Bica, L. Vékás, M. V. Avdeev, O. Marinica, V. Socoliuc, M. Balasoiu and V. M. Garamus, J. Magn. Magn. Mater. 311, 1721 (2007).

15 C. Magnet, P. Kuzhir, G. Bossis, A. Meunier, L. Suloeva and A. Zubarev, Phys. Rev. E 86, 011404 (2012).

16 R. E. Rosensweig, Ferrohydrodynamics (Cambridge University Press, Cambridge, 1985).

17 H. Gavilan, A. Kowalski, D. Heinke, A. Sugunan, J. Sommertune, M. Varon, L. K. Bogart, O. Posth, L. Zeng, D. GonzálezAlonso, Ch. Balceris, J. Fock, E. Wetterskog, C. Frandsen, N. Gehrke, C. Gruttner, A. Fornara, F. Ludwig, S. VeintemillasVerdaguer, C. Johansson and M. P. Morales, Part. Part. Syst. Charact. 34, 1700094 (2017).

18 D. Jiles, Introduction to magnetism and magnetic materials. CRC press, 2015.

19 M. P. Morales, C. J. Serna, F. Bodker and S. Morup, J. Phys.: Condens. Matter 9, 5461-5468 (1997).

20 A.-L. Biance, C. Clanet and D. Quéré, Phys. Fluids 15, 1632 (2003).

21 Y. Pomeau, M. Le Berre, F. Celestini and T. Frisch, C. R. Mec. 340, 867 (2012).

22 L. Maquet, M. Brandenbourger, B. Sobac, A.-L. Biance, P. Colinet and S. Dorbolo, Europhysics Letters 110, 24001 (2015).

23 A.-L. Biance, F. Chevy, C. Clanet, G. Lagubeau and D. Quéré,
J. Fluid Mech. 554, 47-66 (2006).

24 F. Celestini and G. Kirstetter, Soft Matter. 8, 5992-5995 (2012). 